\title{
SUR LA CONCEPTION DES CENTRIFUGEUSES GEOTECHNIQUES
}

\author{
J. Nicolas Font \\ ACUTRONIC France
}

\begin{abstract}
The aim of the paper is to provide the concept principles applied by ACUTRONIC for the design its geotechnical centrifuges range. These designe principles, commonly used by the different geotechnical ACUTRONIC centrifuges, are illustrated by the obtained results from the 665-1 centrifuge model. A list of centrifuge, installed over the world on which geotechnical studies have been performed, is given.
\end{abstract}

\section{RESUME}

Le propos de cette publication est de souligner les principes de conception appliqués par ACUTRONIC dans la réalisation de sa gamme de centrifugeuses géotechniques. Ces principes, communs à tous les modèles de centrifugeuses géotechniques ACUTRONIC, sont illustrés par les résultats obtenus sur la centrifugeuse modèle 665-1. Une liste des centrifugeuses, dans le monde, sur lesquelles des essais géotechniques ont été effectués est fournie.

\section{GENERALITES}

Le but des centrifugeuses est de répondre aux besoins grandissants que manifestent les ingénieurs de mécanique des sols sur la possibilité d'effectuer des essais sur des modèles à échelle réduite. Les mesures effectuées sur ces modèles pouvant être extrapolées à l'échelle 1 au moyen de règles de similitude connues.

Le principe même de ces essais consiste donc à simuler un champ de gravité le plus voisin possible du champ de gravité terrestre (en direction et uniformité) dont l'intensité est égale, à un coefficient près, à l'intensité de ce dernier. Afin d'effectuer leurs essais dans de bonnes conditions expérimentales, les géotechniciens souhaitent donc:

- soumettre un modèle à un champ d'accélération quasi uniforme.

- générer ce champ de façon douce en éliminant d'éventuelles vibrations nuisibles au modèle et à la cohérence de l'expérience.

- pouvoir effectuer des mesures sur le modèle pendant qu'il est soumis au champ d'accélération.

- pouvoir visualiser le modèle.

- pouvoir amener des fluides au niveau du modèle.

- pouvoir préparer le modèle en dehors de la centrifugeuse. 
- pouvoir mettre en place aisément le modèle sur la centrifugeuse.

- disposer sur la centrifugeuse un sytème d'équilibrage aisément mis en œuvre.

une conception rustique et sûre de la centrifugeuse afin d'en limiter l'entretien.

- obtenir une précision et une stabilité de l'intensité du champ d'accélération généré de l'ordre de $1 \%$ de la valeur souhaitée.

- l'optimisation de la puissance nécessaire au fonctionnement de la centrifugeuse.

- assurer un bon antiparasitage électrique des lignes transportants des signaux de bas niveau (mesures, vidéo, ...).

\section{REPONSE D'ACUTRONIC}

Reprenant point par point les souhaits des utilisateurs potentiels, et en s'appuyant sur l'illustration donnée par la figure 1, ci-dessous, ACUTRONIC à concu sa série de centrifugeuses destinées aux essais géotechniques.

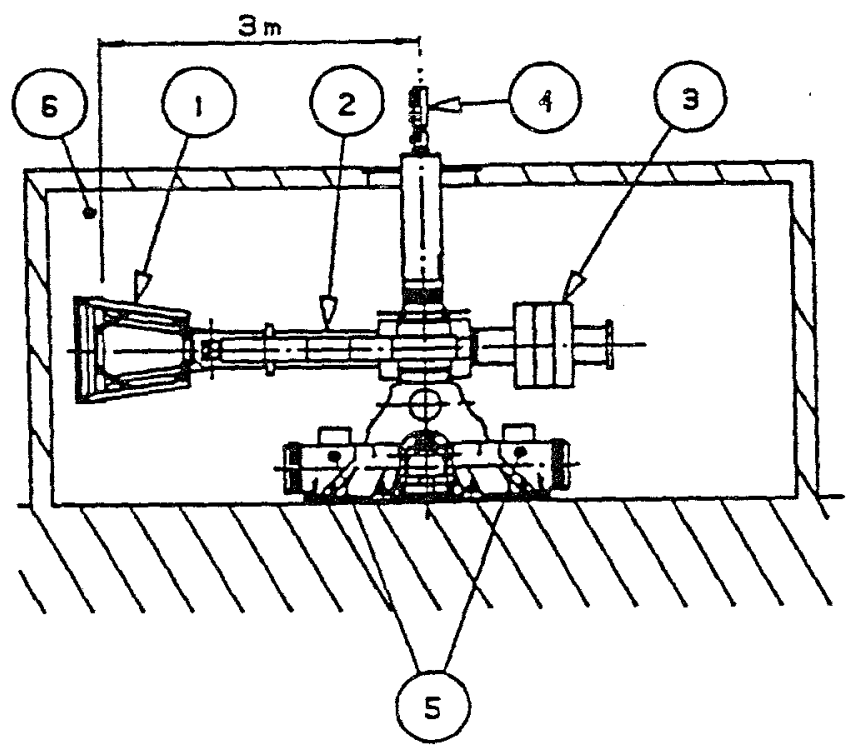

Figure 1. Centrifugeuse Acutronic 665-1

Ces centrifugeuses et la taille maximale du modèle sont dimensionnées suivant les principes décrits par A. N. Schofield (1980) de façon à ce que l'influence de la non uniformité du champ d'accélération généré n'atteigne pas $5 \%$ de la contrainte moyenne subie par le modèle. 
Comme le montre J. Nicolas Font (1988), le faible coefficient de frottement de l'articulation de la nacelle pendulaire (repère 1) contenant le modèle à tester permet d'obtenir une bonne perpendicularité entre le champ d'accélération généré et le plan de pose du modèle. la plus grande des composantes tangentielles générées sur ce plan de pose étant de l'ordre de $1,5 \%$ de la composante normale.

La mise en route de ces centrifugeuses se fait suivant les modes:

- manuel,

- automatique par calculateur (option).

Le fonctionnement en mode manuel se fait par laction sur des roues codeuses permettant d'agir sur la consigne de vitesse de rotation de la machine. Celle-ci exécute les variations de vitesse commandées suivant une rampe électronique faisant vanner progressivement la consigne de vitesse. Dans ce mode, les phases d'accélération ou de décélération de la centrifugeuse se font à $\mathrm{dw} / \mathrm{dt}$ constant.

Le fonctionnement en mode automatique se fait par l'intermédiaire d'un calculateur de pilotage. Avant le démarrage de la machine l'utilisateur programme le profil d'accélération souhaité en fonction du temps. Lors du fonctionnement de la machine, le calculateur donne à tout moment la vitesse de rotation correspondant à l'accélération souhaitée et corrige la consigne de vitesse si nécessaire. Dans ce mode les phases d'accélération ou de décélération de la centrifugeuse se font à $\mathrm{dG} / \mathrm{d} t$ constant. La mise en vitesse la plus rapide de ces centrifugeuses permet d'atteindre l'accélération centrifuge de $100 \mathrm{~g}$ en $180 \mathrm{~s}$ et $200 \mathrm{~g}$ en $360 \mathrm{~s}$. L'accélération tangentielle maximale engendrée lors des phases d'accélération et de décélération est de l'ordre de $2 \mathrm{~g}$. L'intensité efficace des vibrations existantes au niveau du modèle est de l'ordre de $0,1 \mathrm{~g}$.

Les centrifugeuses ACUTRONIC sont équipées de contacts tournants de très grande qualité permettant de relier électriquement et sans parasite la partie mobile et la partie fixe de la centrifugeuse. Ces contacts toumants permettent donc d'établir les liaisons nécessaires aux signaux issus des appareils de mesure et à l'alimentation en puissance de la partie tournante. Le nombre des contacts est défini avec le client en fonction des besoins exprimés. En règle générale le nombre de contacts tournants est voisin de 100 . Il est cependant conseillé d'amplifier les signaux de très bas niveau issus des appareils de mesure avant de traverser les contacts tournants. 
En option, ces centrifugeuses peuvent être équipées d'un système de joint tournant fibre optique permettant d'établir une liaison "fullduplex à haute fréquence (jusqu'à $50 \mathrm{Mbits} / \mathrm{s}$ ).

En option, les centrifugcuses ACUTRONIC peuvent être équipées d'un système vidéo permettant de visualiser le modèle pendant l'essai. Les signaux vidéo issus d'une caméra $C C D$, transitent par les contacts tournants et aboutissent au moniteur placé dans la salle de commande. Une autre possibilité déjà réalisée pour KAJMMA Institute consiste dans la synchronisation d'une caméra rapide à la rotation de la centrifugeuse et d'effectuer ainsi des prises de vue au travers de l'ouvernure offerte par le carénage arrière.

Les centrifugeuses ACUTRONIC peuvent être équipées de systèmes de joints toumants (repère 4) permettant des liaisons fluidiques entre la partie tournante et la partie fixe de la centrifugeuse. Ces fluides pelivent être de l'eau, de l'huile, de l'air, du fréon,... Le nombre, la quantité et les conditions de fonctionnement de ces joints tournants sont définis avec le client.

D'une facon générale la préparation du modèle demande un grand nombre d'heures de travail. Aussi pour ne pas immobiliser la centrifugeuse, les modèles sont construits en dehors de la machine. La préparation et l'instrumentation de deux modèles simultanément paraît aussi illusoire. Donc les centrifugeuses ACUTRONIC sont dyssimétriques et ne permettent de tester qu'un seul modèle à la fois. Cette configuration permet d'optimiser la puissance nécessaire à l'entraînement de la centrifugeuse (voir paragraphe 4). De façon à permettre une mise en place aisée du modèle, une large ouverture pratiquée dans le carénage de la nacelle donne un accès direct au plan de pose du container renfermant le modèle. La mise en place de ce dernier n'exige le démontage d'aucune pièce de la centrifugeuse.

Les centrifugeuses ACUTRONIC sont équipées d'un système original de mesure permanente du balourd. Cette mesure est intégrée au système de gestion de la sécurité de la machine. Cette mesure est aussi utilisée pour déterminer la position d'équilibrage des contrepoids de masse fixe (repère 3). L'équilibrage de ces centrifugeuses s'obtient donc par le déplacement à l'arrêt d'une masse fixe le long du bras de la centrifugeuse.

En option (voir paragraphe 5) ce système est complété par un système breveté d'équilibrage automatique pendant la rotation de la machine.

Les pièces les plus sollicitées d'une centrifugeuse sont ses bras (repère 2). Afin de s'assurer d'une bonne qualité et d'un bon vieillissement dans le temps, ACUTRONIC a choisi une structure monolytique pour les bras 
de ses centrifugeuses. Les deux tubes constituant le bras, réalisés d'un seul tenant, relient les contrepoids à la nacelle contenant le modèle testé. Ces bras sont obtenus par forage de ronds forgés de grande qualité métallurgique. Côté contrepoids un filetage est usiné ; il permet leur déplacement. L'entretien de la machine se réduit dès lors à la lubrification périodique de certains éléments mécaniques (roulements, réducteur, ...).

De la mesure de vitesse de la centrifugeuse, à partir d'un codeur optique donnant plusieurs milliers d'impulsions par tour une boucle d'asservissement en vitesse de précision est réalisée qui permet d'obtenir le degré de précision et de stabilité souhaité.

Comme le montre J. Nicolas Font (1988) la puissance nécessaire à la rotation des centrifugeuses est surtout due aux pertes aérodynamiques occasionnées par la vitesse périphérique de la nacelle.

Aussi cette nacelle possède des carénages aérodynamiques et les centrifugeuses tournent dans une enceinte circulaire (repère 6) dimensionnée de facon à optimiser la puissance nécessaire. A ce sujet, voir paragraphe 4.

Afin de minimiser les parasites électriques issus de la génération de puissance, l'entrainement des centrifugeuses se fait par des moteurs électriques à courant alternatif. Pour des questions de coût l'ancienne version de motorisation employée pour la centrifugeuse 680 du LCPC (moteur à vitesse constante + embrayage - frein à courant de Foucault) a été abandonnée au profit de la variation de vitesse par variation de fréquence (repère 5). De façon à réduire l'encombrement et le coût, la chaîne de motorisation de toutes les centrifugeuses comprend un ou plusieurs moteurs à grande vitesse $(1000,1500$ ou $1800 \mathrm{tr} / \mathrm{mn})$ accouplés à un renvoi d'angle. Un filtrage efficace et une large séparation physique des circuits électriques de bas niveaux et de puissance éliminent pratiquement tout effet de parasitage transmis par conduction ou par rayonnement.

\section{PERFORMANCES D'UNE CENTRIFUGEUSE}

Les performances d'une centrifugeuse sont définies par :

- son rayon nominal : distance entre l'axe de rotation et le centre de gravité du modèle à tester.

- les dimensions du modèle à tester.

- le domaine d'utilisation : diagramme donnant l'accélération centrifuge possible en fonction de la masse du modèle tester. 
Pour la centrifugeuse $665-1$, le rayon nominal est de $2,7 \mathrm{~m}$. Les dimensions maximales du modèle à tester sont $1000 \mathrm{~mm}$ x $800 \mathrm{~mm}$ x $800 \mathrm{~mm}$.

Son domaine d'utilisation est donné par la figure 2.

Ce domaine est délimité par :

1. Limitation en accélération due à la vitesse maximale du moteur.

2. Limitation en masse due à la capacité d'équilibrage des contrepoids.

3. Limitation en accélération et masse due aux contraintes dans la structure de la machine.

Les autres spécifications de la centrifugeuse modèle 665-1 sont :

- Planeité du plan de pose du modèle :

- Sans charge

- Sous charge max.

- Balourd maximal accepté

- Puissance électrique

- Temps de montée à $100 \mathrm{~g}$

- Temps de montée à $200 \mathrm{~g}$ $\pm 0,2 \mathrm{~mm} \max$.

$\pm 0,5 \mathrm{~mm} \max$.

$\pm 50000 \mathrm{~N}$.

$200 \mathrm{kVA}$.

$3 \mathrm{~mm}$.

$6 \mathrm{~mm}$.

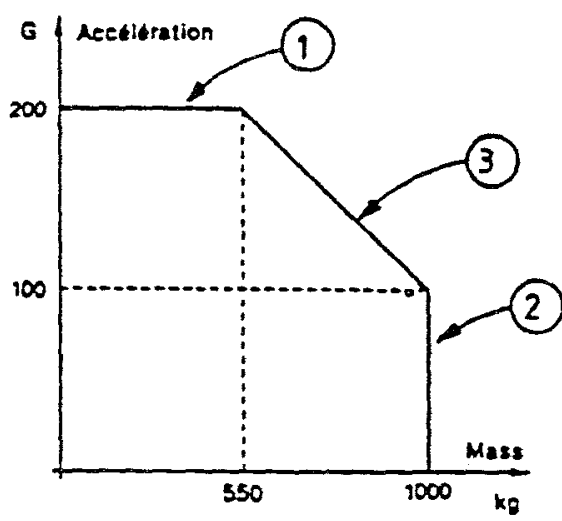

Figure 2. Domaine d"utilisation modèle 665-1.

\section{AERODYNAMIQUE}

L'étude aérodynamique de la centrifugeuse et de son enceinte est très importante car elle conditionne la puissance nécessaire à sa rotation ainsi que la surpression que la rotation de la machine génère dans cette enceinte. 


\subsection{Pertes aérodynamiques.}

La géométrie des centrifugeuses géotechniques ACUTRONIC est telle que les pertes aérodynamiques causées par la nacelle sont largement prépondérantes devant celles provoquées par les autres éléments de la partie tournante. Ceci n'est pas vrai pour les centrifugeuses à bras symétriques pour lesquelles le calcul montre un accroissement de la puissance consommée supérieure à 1,5 fois pour des dimensions géométriques équivalentes. Le schéma aérodynamique adopté pour le calcul de ces pertes consiste à considérer la nacelle comme un solide de section $S_{n}$ se déplaçant dans un tunnel de section $S_{0}$ à la vitesse $\left(R w-V_{v}\right)$.

où $R$ = rayon nominal de la centrifugeuse

$w=$ vitesse angulaire de la centrifugeuse

$\mathrm{V}_{\mathrm{v}}=$ vitesse absolue de l'air dans l'enceinte.

La Figure 3 illustre ce schéma. L'étude théorique montre qu'il existe une relation linéaire entre $R w$ et $V v$ et l'on peut écrire:

$$
\mathrm{V}_{\mathrm{v}}=\mathrm{K}_{\mathrm{v}} \mathrm{Rw}
$$

La puissance dissipée par les pertes aérodynamiques s'écrit :

$$
P=\frac{1}{2} \rho S_{n} C_{x e}\left(R w-V_{v}\right)^{2} R w
$$

ou

$$
P=\frac{1}{2} \rho S_{n}(R w)^{3} C_{x e}\left(1-K_{v}\right)^{2}
$$

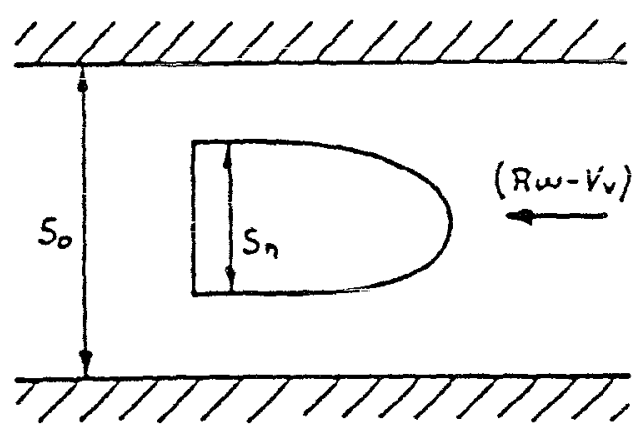

Figure 3. Schéma aérodynamique.

A la pression atmosphérique, $\mathrm{C}_{\mathrm{xe}}$ et $\mathrm{K}_{\mathrm{V}}$ sont fonction de :

- $\mathrm{C}_{\mathrm{xi}}$ : coefficient de trainée de la nacelle en écoulement infini.

$-S_{\mathrm{n}} / \mathrm{S}_{\mathrm{O}}$.

- l'état de surface des parois de l'enceinte. 
La fugure 4 donne l'évolution du $K_{V}$ et du coefficient de puissance $C_{x e}\left(1-K_{V}\right)^{2}$ dans le cas d'une centrifugeuse équipée d'une nacelle dont $C_{x i}=0,35 ;$ en fonction du rapport $S_{\sqrt{n}} / S_{0}$.

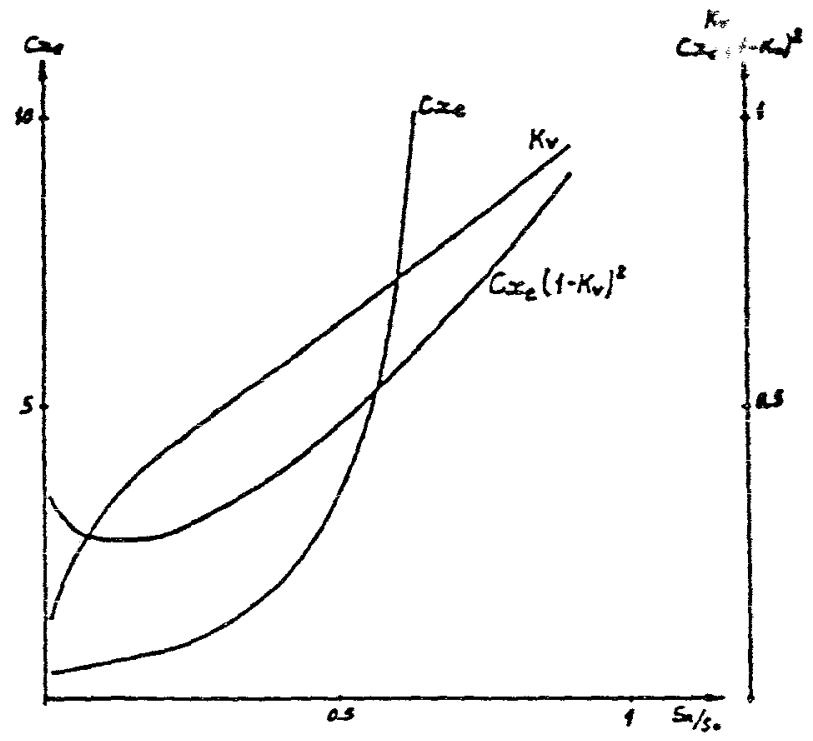

Figure 4. Puissance avec une nacelle.

La figure 5 donne l'évolution du $\mathrm{K}_{\mathrm{y}}$ et du coefficient de puissance $\mathrm{C}_{\mathrm{xe}}(1-\mathrm{Kv})^{2}$ dans le cas d'une centrifugeuse équipée de deux nacelles, les autres paramètres restant identiques.

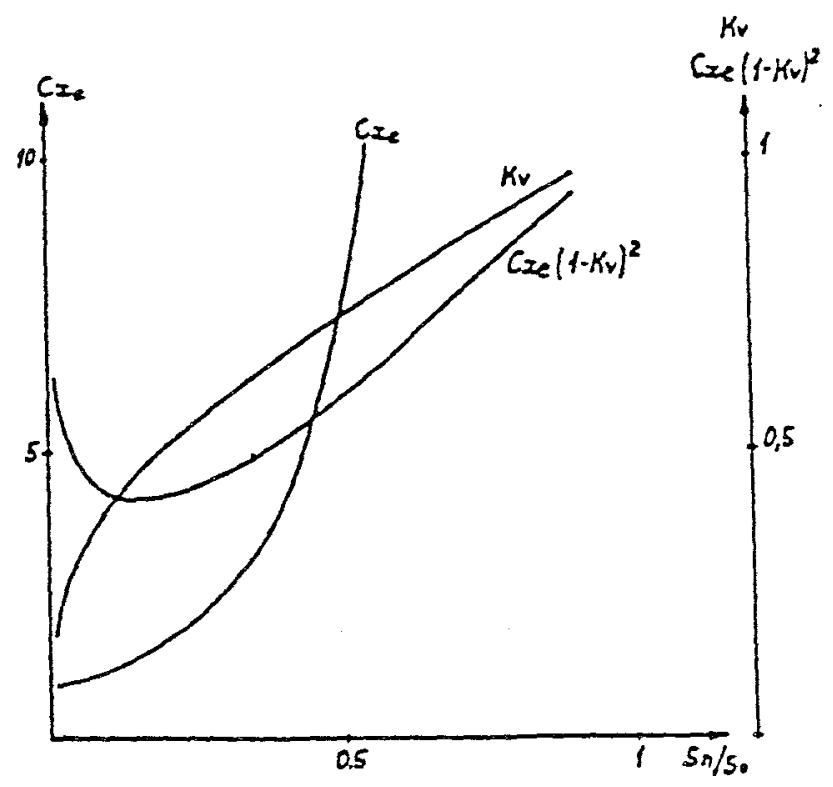

Figure 5. Puissance avec deux nacelles. 
Dans les deux cas, le coefficient de puissance $C_{x e}\left(1-K_{v}\right)^{2}$ passe par un optimum, ceci montre l'impontance du dimensionnement de l'enceinte.

Pour cet optimum, le fait de rajouter une deuxième nacelle à la centrifugeuse, multiplie par 1,5 la puissance nécessaire à son entrainement.

Pour la centrifugeuse $665-1$, on a :

\begin{tabular}{|c|c|}
\hline$C_{x i}$ & $=1,141$ \\
\hline$S_{n}$ & $=1,05 \mathrm{~m}^{2}$ \\
\hline$S_{0}$ & $=3,9 \mathrm{~m}^{2}$ \\
\hline$C_{x e}$ & $=3,801$ \\
\hline $\mathrm{K}_{\mathrm{v}}$ & $=0,653$ \\
\hline$C_{x e}\left(1-K_{y}\right)^{2}$ & $=0,458$ \\
\hline
\end{tabular}

La figure 6 donne la courbe de puissance nécessaire à l'entrainement de la machine pendant les régimes à vitesse de rotation constante.

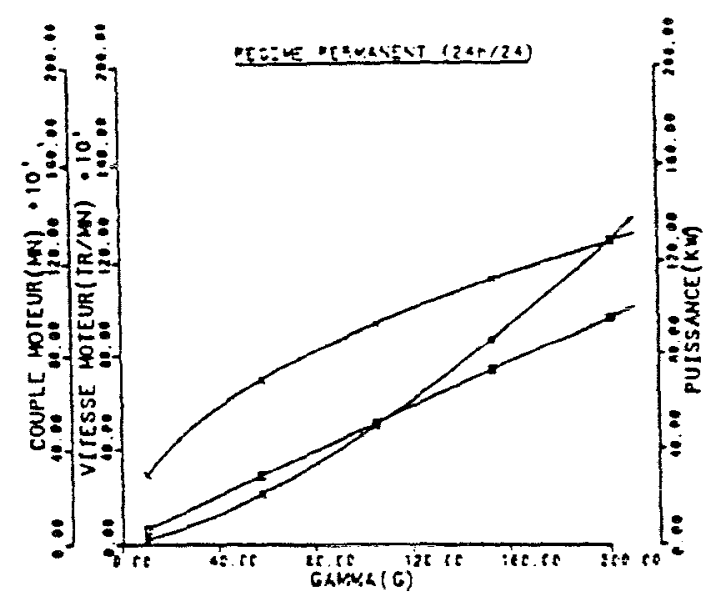

Figure 6. Puissance à vitesse constante.

La figure 7 donne la puissance nécessaire à l'entrainement de la machine pendant les phases d'accélération et de freinage entre 0 et $200 \mathrm{~g}$.

Les pertes aérodynamiques sont équivalentes à des calories qui réchauffent l'air contenu par l'enceinte de la centrifugeuse. Afin de maintenir la variation de température dans l'enceinte, dans des limites raisonnables $\left(10^{\circ} \mathrm{C}\right.$ par rapport à la température extérieure), il y a lieu de prévoir un système de ventilation. Une étude sur modèle réduit a été menée. Elle a montré qu'il était nécessaire de prendre des précautions sur la réalisation de cette ventilation. En effet, suivant la disposition des trous de ventilation dans l'enceinte, celle-ci peut 
accroître les pertes aérodynamiques de facon sensible (environ $20 \%$ ) car elle augmente le débit de fuite de l'enceinte. Une configuration optimale a été définie et elle est appliquée sur toutes les installations ACUTRONIC. La centrifugeuse $665-1$ nécessite un débit d'air de ventilation de $8 \mathrm{~m}^{3} / \mathrm{s}$.

\subsection{Surpression dans l'enceinte.}

Pour calculer la surpression que génère la rotation de la centrifugeuse à l'intérieur de l'enceinte on conserve le même schéma aérodynamique que pour le calcul de puissance, en considérant que l'enceinte est étanche. Ce schéma permet seulement de calculer la surpression moyenne ou stationnaire. Le calcul s'effectue en considérant dans l'enceinie deux zones distincies :

- la première s'étendant depuis l'axe de rotation de la centrifugeuse jusqu'au voisinage de la nacelle oì la surppression est provoquée par la rotation de l'air.

- la deuxième s'étendant depuis la frontière de la première jusqu'au mur vertical de l'enceinte où se superpose, à la surpression provoquée par la rotation de l'air, la pression générée par le déplacement de la nacelle dans son tunnel.

Si l'on appelle DP1 la surpression provoquée par la rotation de l'air, et DP2 la surpression provoquée par le déplacement de la nacelle dans son tunnel, on obtient dans l'hypothèse des gaz incompressibles :

$$
\begin{aligned}
& D_{\mathrm{P} 1}=101330 \mathrm{e}^{\frac{\mathrm{K}_{v}^{2} w^{2}}{\Gamma T} \frac{\mathrm{R}^{2}}{2}} \\
& \mathrm{D}_{\mathrm{P} 2}=\frac{1}{2} \rho \mathrm{w}^{2}\left[\delta \mathrm{R}^{2}\left(1-\mathrm{K}_{v}\right)^{2}+\mathrm{K}_{v}^{2} \mathrm{R}^{2}\right]
\end{aligned}
$$

La surpression est donc égale à:

$$
\begin{aligned}
& \text { - } \mathrm{DP}=\mathrm{D}_{\mathrm{pl}} \quad \text { dans la première zone } \\
& -\mathrm{DP}=\mathrm{D}_{\mathrm{p} 1}+\mathrm{D}_{\mathrm{p} 2} \quad \text { dans la deuxième zone. }
\end{aligned}
$$

Tous les calculs faits, la figure 8 donne la surpression moyenne dans l'enceinte de la centrifugeuse 665-1. Cette surpression se développe sur toutes les parois de l'enceinte et les effets qu'elle génère sont supportés par celles-ci et la porte d'accès de l'enceinte.

Les mesures de pression effectuées dans l'enceinte de la centrifugeuse 680 (LCPC) montrent l'existence d'une onde de pression instationnaire. Le déplacement de cette onde est synchronisé avec la rotation du bras de la machine. 
Cette onde génère à son tour une surpression qui se superpose à la surpression moyenne. Les mesures permettent d'estimer que la surpression instationnaire est du même ordre de grandeur que la surpression moyenne. Il existe donc dans l'enceinte une surpression cyclique instantanée dont l'intensité est le double de la pression moyenne. Ainsi, pour la centrifugeuse 665-1, la surpression moyenne maximale (sur le mur vertical) est de $5350 \mathrm{~Pa}$, la surpression instantanée est de $10700 \mathrm{~Pa}$ et cette dernière excite la structure de l'enceinte à la fréquence de rotation de la centrifugeuse, soit $4,3 \mathrm{~Hz}$ à $200 \mathrm{~g}$.

\section{L'EQUILIBRAGE AUTOMATIQUE}

En option, toutes les centrifugeuses géotechniques ACUTRONIC peuvent être équipées du système d'équilibrage automatique qui fait l'objet d'un brevet. Le système standard de contrepoids déplaçables à l'arrêt permet d'équilibrer la masse maximale utile, soit $1000 \mathrm{~kg}$ pour la centrifugeuse 665-1.

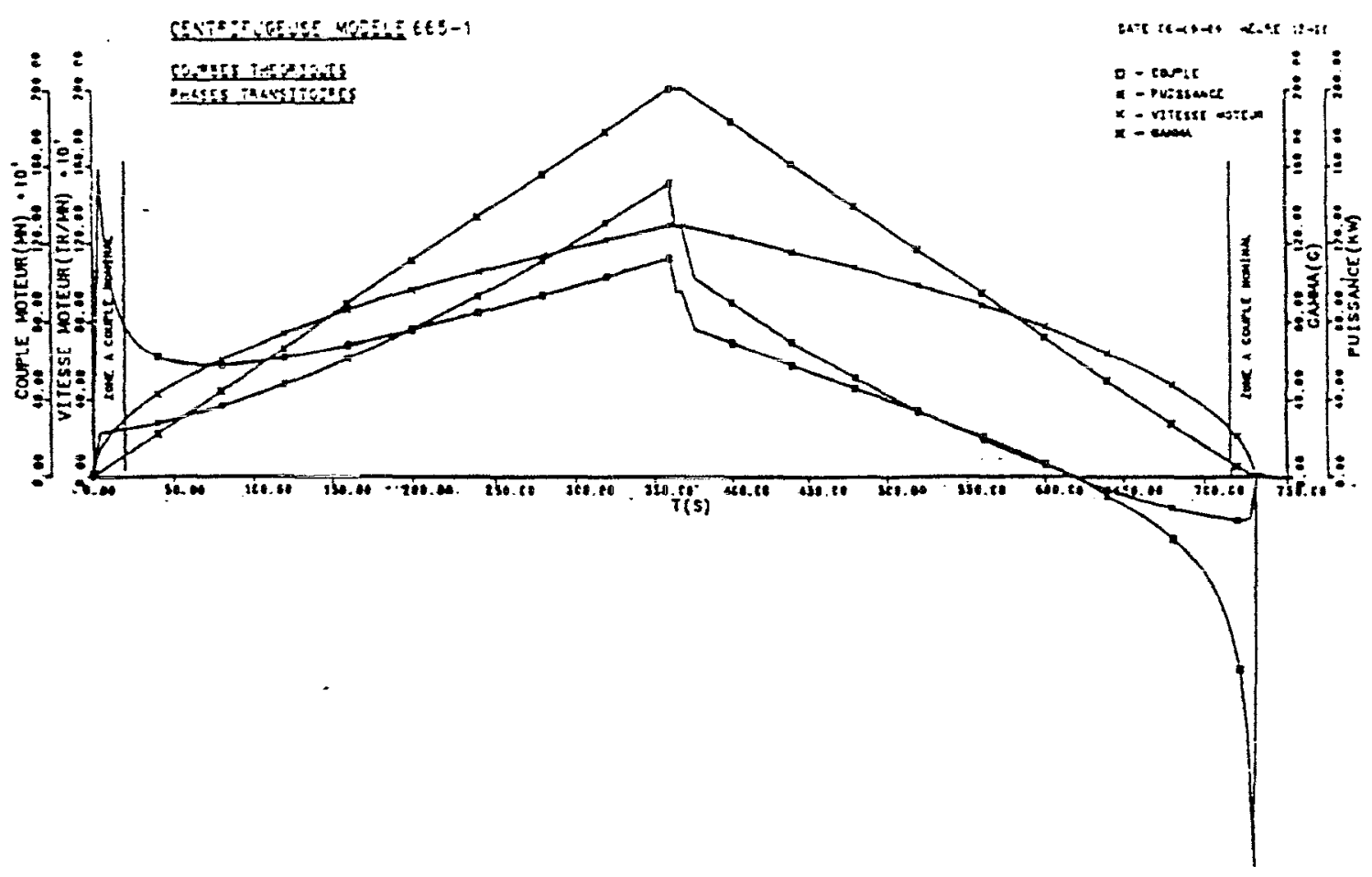

Figure 7. Puissance pendant les phases d'accéleration et de freinage entre 0 et $200 \mathrm{~g}$. 


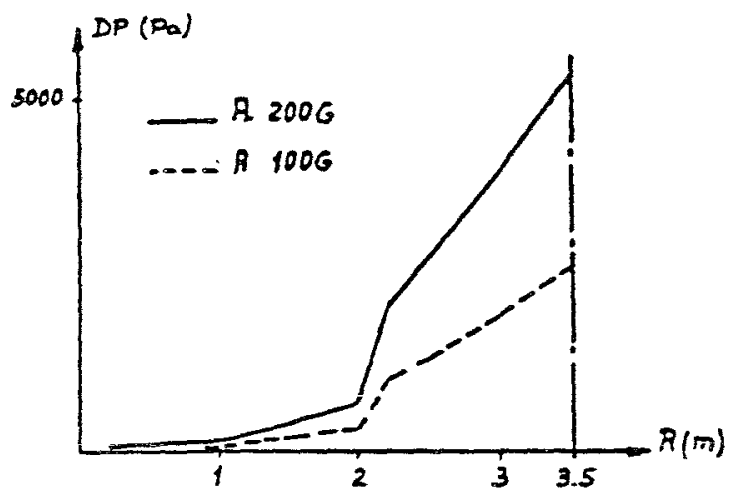

Floure 8. Surpression dans l'enceinte.

Le système d'équilibrage automatique est complémentaire au système standard et il permet de compenser des petites variations de masse ou de déplacement du centre de gravité du modẻle.Pour des raisons de sécurité, le système d'équilibrage automatique peut uniquement compenser le balourd maximal acceptable pour la machine.

Figure 9. Equilibrage automatique.

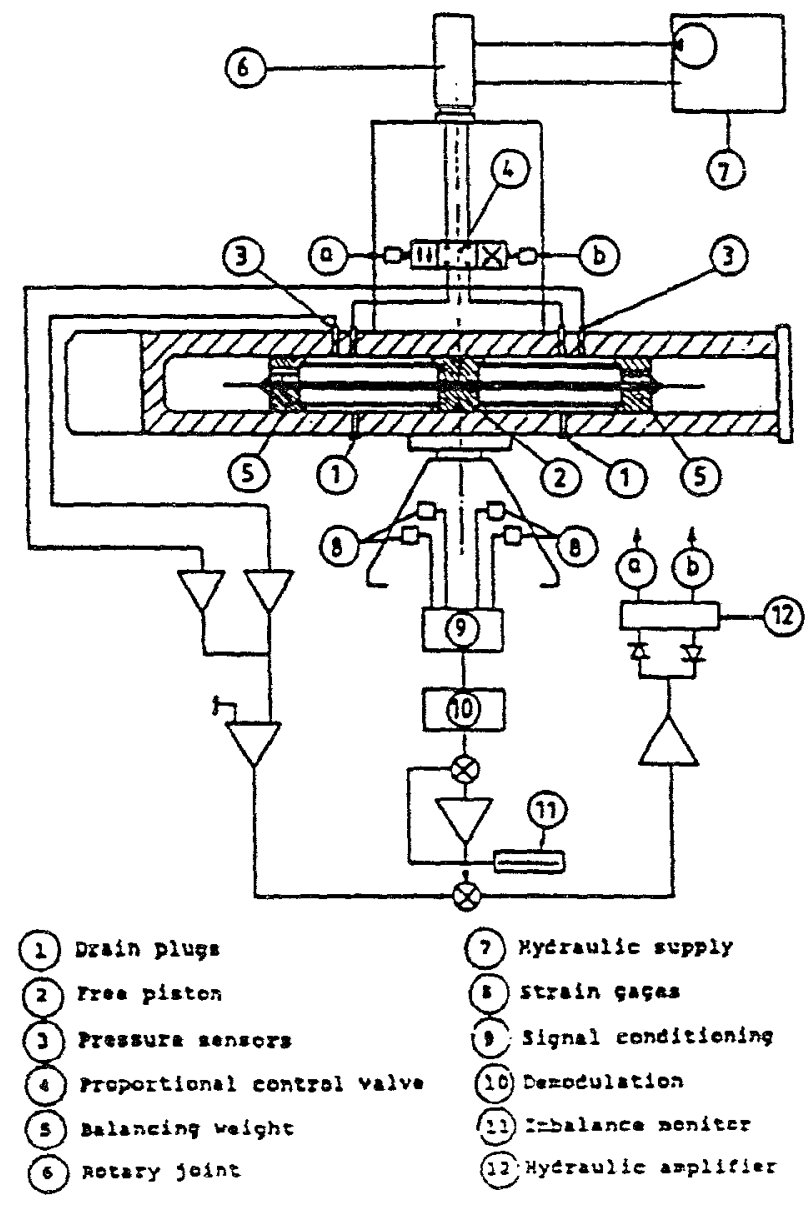


Le principe de ce système (voir figure 9) repose sur l'existence, à l'intérieur de chaque bras, d'un piston mobile asservi au signal fourni par le système de mesure de balourd. Ces pistons se déplacent grâce à la pression fournie, par une centrale hydraulique, dimensionnée en débit de facon à ce que le déplacement maximal du piston s'effectue en une minute.

Le déplacement de chaque piston est commandé par une électrovanne proportionnelle dont le signal de commande est fourni par le système standard de mesure de balourd équipant la centrifugeuse. Le mouvement des pistons s'arrête lorsque la mesure de balourd donne un signal nul.

\section{SUR LA MESURE DU BALOURD}

Comme le montre J. Nicolas Font (1988) les contrepoids permettent seulement l'équilibrage statique des centrifugeuses dissymétriques. Cet équilibrage serait parfait si le centre de gravité de la nacelle et de la charge se trouvait, lors de la rotation de la machine, dans le prolongement de l'axe des bras comme cela est le cas pour le centre de gravité des contrepoids. Hormis l'accélération centrifuge la nacelle et la charge sont soumis à l'accélération de la pesanteur. La conjugaison de cette dernière et de l'existance de frottement dans la rotule d'articulation font que le centre de gravité de la nacelle et de la charge ne se trouvent jamais dans la position idéale.

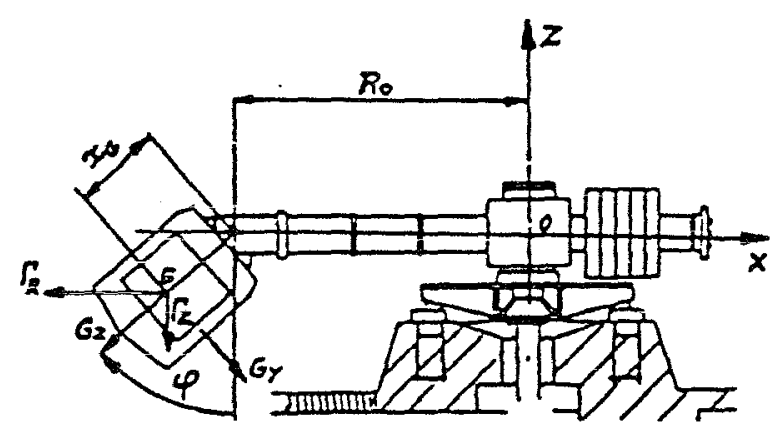

Figure 10. Incinaison de ia nacelle.

Si l'on désigne par $\varphi$ l'angle d'inclinaison de la nacelle (figure 10), il est aisé de montrer qu'il existe un moment autour du point $\mathrm{O}$ tel que :

$$
\text { Moy }=-m \Gamma_{R} x_{G} \cos \varphi
$$


Si D $\varphi$ est "l'erreur" de position de la nacelle, l'expression de ce moment devient :

$$
\operatorname{Moy}=-m \Gamma_{\mathrm{R}} \mathrm{x}_{\mathrm{G}} \sin \varphi
$$

L'expérience montre que $\mathrm{D} \phi$ est sensiblement constant et égal a 1,5 degrés à partir de $\Gamma_{R}=10 \mathrm{~g}$, aussi Moy varie linéairement avec $\Gamma_{\mathrm{R}}$.

Ce moment ne peut être compensé par les contrepoids que si le centre de gravité de ceux-ci se trouve à la même hauteur z que le centre de gravité de la nacelle. Ce résultat ne peut être obtenu que par une centrifugeuse parfaitement symétrique, donc avec deux nacelles.

L'intensité de ce moment n'est pas un facteur dimensionnant de la pivoterie de la centrifugeuse.

Dans le cas de la ceñuifugeuse 680, sa structure pemet de décoüpler (au niveau de la mesure) l'effort de balourd suivani $x$ du moment Moy. Lorsque les contrepoids se trouvent à :

$$
\mathrm{x}_{\mathrm{cp}}=\frac{\mathrm{m}\left(\mathrm{x}_{\mathrm{G}}+\mathrm{Ro}\right)}{\mathrm{CP}}
$$

Le signal donné alors par le système de mesure de balourd est bien nul. Dans le cas des centrifugeuses 661 et 665 , leur structure ne permet pas de découplage entre l'effort de balourd et le moment Moy : cette mesure se fait par l'intermédiaire des contraintes existantes dans l'embase de ces machines.

Le signal de balourd est donc influencé par le moment Moy. L'étalonnage de la chaîne de mesure de balourd permet de quantifier l'influence du moment et d'en tenir compte dans la formule donnant le déplacement du contrepoids. Le système d'équilibrage automatique minimise, en fait, les contraintes dans l'embase des machines.

\section{SIMULATION DYNAMIQUE}

De plus en plus, les ingénieurs en mécanique des sols souhaitent réaliser des essais dynamiques sur centrifugeuses : simuler des tremblements de terre.

Si l'échelle du modèle testé sur centrifugeuse est $1 / \mathrm{N}$, les conditions de similitude impliquent que, par rapport au modèle à l'échelle 1 , il faut multiplier l'accélération et la fréquence du phénomène simulé par le coefficient $\mathrm{N}$, HonYim Ko (1988). Dans la nature, les excitations provoquées par les tremblements de terre ont trois composantes : deux horizontales et une verticale. L'intensité 
d'accélération sur ces trois composantes peut varier de 0 à $0,5 \mathrm{~g}$ et la gamme de fréquence de 1 à $10 \mathrm{~Hz}$.

Il est clair que sur un modèle à l'échelle $1 / 100$, soumis à une accélération centrifuge de $100 \mathrm{~g}$, la similitude implique que les excitations devront avoir une intensité d'accélération comprise entre 0 et $50 \mathrm{~g}$ et une gamme de fréquence de 100 à $1000 \mathrm{~Hz}$.

Rapidement il apparaît que la composante verticale (parallèle aux bras de la centrifugeuse) est impossible à réaliser car l'effor nécessaire à l'excitation est très grand parce qu'il doit faire subir à la masse excitée une accélération maximale de $150 \mathrm{~g}$. En outre cet effort dépasse l'effort de balourd maximal toléré.

Par contre, moyennant certaines précautions il est possible d'effectuer des excitations dans le plan parallèle au plan de pose du modèle dans la nacelle. La première précaution à prendre est de découpler au mieux les efforts d'excitation de la structure de la centrifugeuse. Ce découplage peut être obtenu par un dessin adéquat des supports de la plateforme de la nacelle (faible raideur dans la direction d'excitation) et par l'utilisation de composants mécaniques à faible coefficient de frottement pour la réalisation de l'articulation entre la nacelle et les bras.

L'effort maximum, causé par l'excitation, pouvant être appliqué à l'extrémité du bras (verticalement ou horizontalement) est limité par le niveau de contrainte qu'il génère dans la structure de la centrifugeuse. L'existence dans la structure de résonances mécaniques à certaines fréquences provoque l'amplification des contraintes. A ces fréquences, les forces d'excitation doivent être diminuées de manière à conserver des niveaux de contrainte acceptables. Cependant, les centrifugeuses possèdent des fréquences de résonance dangereuses (avec grand coefficient de surtension) aux basses fréquences : inférieures à $20 \mathrm{~Hz}$, en dehors du domaine de fréquence de simulation des tremblements de terre.

Le découplage de la masse excitée (mobile) par rapport à la plateforme de la nacelle est aisément réalisé par l'utilisation de tables à butées à huile, ou mieux avec des butées à air comme ACUTRONIC l'a réalisé pour la centrifugeuse de l'Université de Princeton.

Un des principaux problèmes posé par la simulation des tremblements de terre sur centrifugeuse est le moyen à utiliser pour développer l'effort d'excitation. Les excitateurs électriques sont à bannir car leur taille et leur masse deviennent considérables. En effet l'excitation d'une masse de $400 \mathrm{~kg}$ à $100 \mathrm{~Hz}$ 
avec une accélération de $50 \mathrm{~g}$ nécessite une puissance d'environ $400 \mathrm{~kW}$. Séduisante parce qu'elle permet de stocker au préalable l'énergie nécessaire, la solution hydraulique a également des limites très restrictives. En effet, sans parler de la force à développer, si l'on considère le haut de gamme des vérins dynamiques que l'on peut trouver, leur vitesse de déplacement est limité à $1 \mathrm{~m} / \mathrm{s}$. En considérant un vérin dont la course totale est de $50 \mathrm{~mm}$ on obtient le domaine d'accélération réalisable, dans un fonctionnement sinusoïdal, donné par la ñgure 11. Ce domaine se décompose en quatre zones correspondant chacune à une limitation technologique. Ces zones sont :

- limitation due à la course du vérin.

- limitation due à la vitesse du vérin.

- limitation due à la force du vérin.

- limitation due à la réponse en fréquence des servovalves.

A ces limitations il faut ajouter le mauvais fonctionnement éventuel des composants hydrauliques lorsqu'ils sont soumis à de grands niveaux d'accélération.

Actuellement, ACUTRONIC explore le moyen d'obtenir les excitations de simulation désirées à partir d'explosifs en collaboration avec le CEA-CESTA.

La description de cette méthode est esquissée par B. Bourdin (1988).

\section{SECURITE}

Les centrifugeuses sont des machines dangereuses il est nécessaire de prendre toutes les dispositions nécessaires pour l'obtention du maximum de sécurité dès leur étude:

- Dimensionnement de toutes les pièces critiques avec un coefficient de sécurité de 3 à la limite élastique, même lorsque le balourd atteint la valeur maximale admissible.

- Dimensionnement de toutes les pièces critiques avec coefficient de sécurité de 1 à la limite élastique en cas de balourd catastrophique (perte de tout le modèle).

- Gestion des sécurités par logique cablée et signalisation au pupitre.

- Mesure permanente du balourd et déclenchement d'une sécurité en cas d'atteinte de la valeur admissible.

- Donner la priorité aux sécurités cablées même lorsque la machine est pilotée par calculateur.

- En option le système de sécurité standard est complété par un système de surveillance vidéo, de mesure des vibrations, de détection de fumée,... 


\section{MODELISATION}

Bien que ce ne soit pas de la compétence des ingénieurs d'ACUTRONIC, la simulation sur modèle réduił en mecanique de sols est analogue à la simulation sur modèle réduit en mécanique des nuides.

Il est bien connu en mécanique des fluides que l'on ne peut, sans danger pour l'interprétation des résultats, réduire indéfiniment la taille des modèles. Après certains échecs, les aérodynamiciens réclament de plus en plus des moyens d'essais permettant d'effectuer les simulations sur des modeles dont l'échelle n'est pas exagérément petite.

Tôt ou tard la mécanique des sols rencontrera les mêmes problèmes (si ce n'est déjà fait) car de plus en plus les utilisateurs potentiels de centrifugeuses souhaitent dces machines développant de grands niveaux d'accélération au détriment de la taille du modèle. Comme toujours il n'existe pas de machine universelle et il faut adapter les performances de celle-ci aux problèmes étudiés :

- centrifugeuse de grande capacité en volume et masse avec une accélération d'environ $100 \mathrm{~g}$ pour l'étude des phénomènes statiques ou de basse fréquence (tremblement de terre).

- centrifugeuse de petite capacité en volume et masse avec une accélération d'environ $400 \mathrm{~g}$ pour l'étude des phénomènes rapides (explosions, pénétration,...).

Les performances des deux machines décrites ci-dessus sont très difficilement conciliables d'un point de vue mécanique et technologique .

Figure 11. Domaine daccélération des verins hydrauliques.

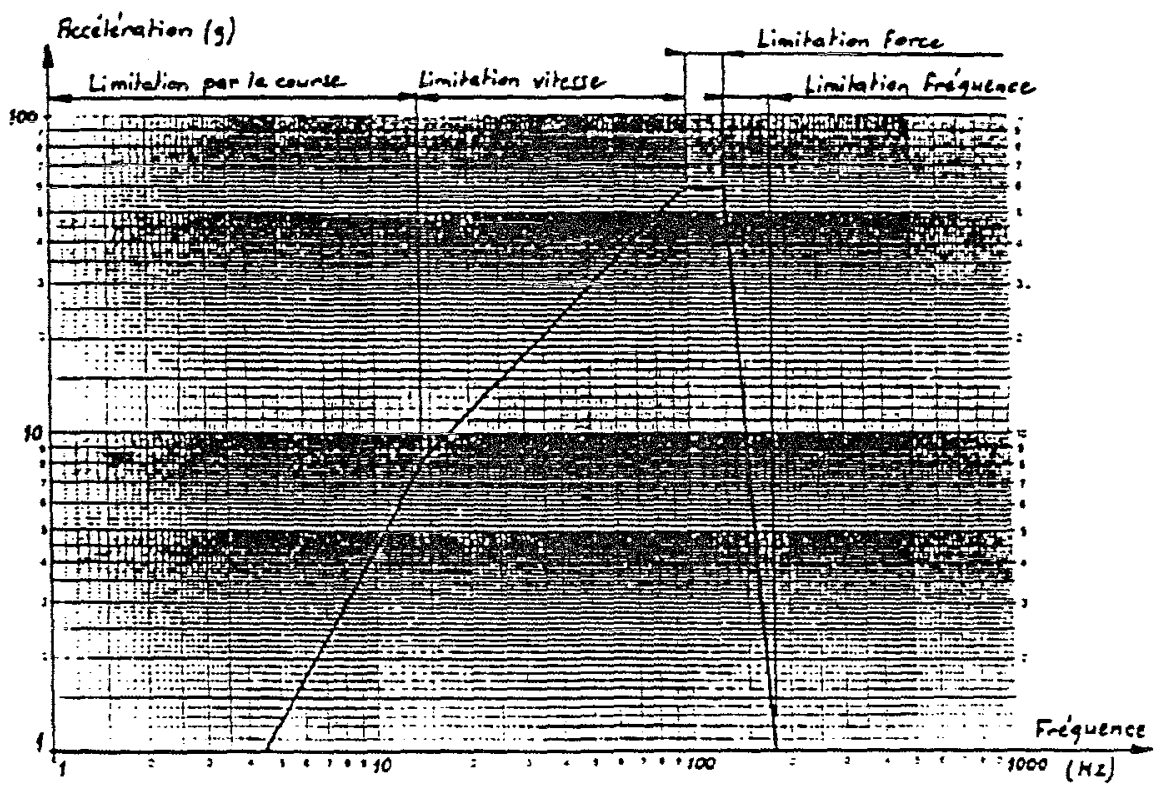




\section{CENTRIFUGEUSES EXISTANTES}

Sans vouloir être exhaustif les tableaux des pages suivantes donnent la liste des centrifugeuses opérationnelles ou en projet actuellement. Cette liste est classée par pays.

L'attention du lecteur est attirée sur le fait que charge maximale et accélération maximale ne vont pas de paire

\section{REFERENCES}

BOURDIN B., Vibrations et simulation des séismes sur fondations en centrifugeuse. Centrifuge 88, Corté 1988 Balkema, Rotterdam.

HON-YIM KO, Summary of the state-of-the-art in centrifuge model testing. Centrifuges in Soil Mechanics, Craig, James and Schofield. 1988 Balkema, Rotterdam.

NICOLAS FONT J., Design of geotechnical centrifuges. Centrifuge 88 , Coné 1988 Balkema, Rotterdam.

SCHOFIELD A. N., Cambridge geotechnical centrifuge operations. Geotechnique, Vol 30, p. $227 \& 268$ 


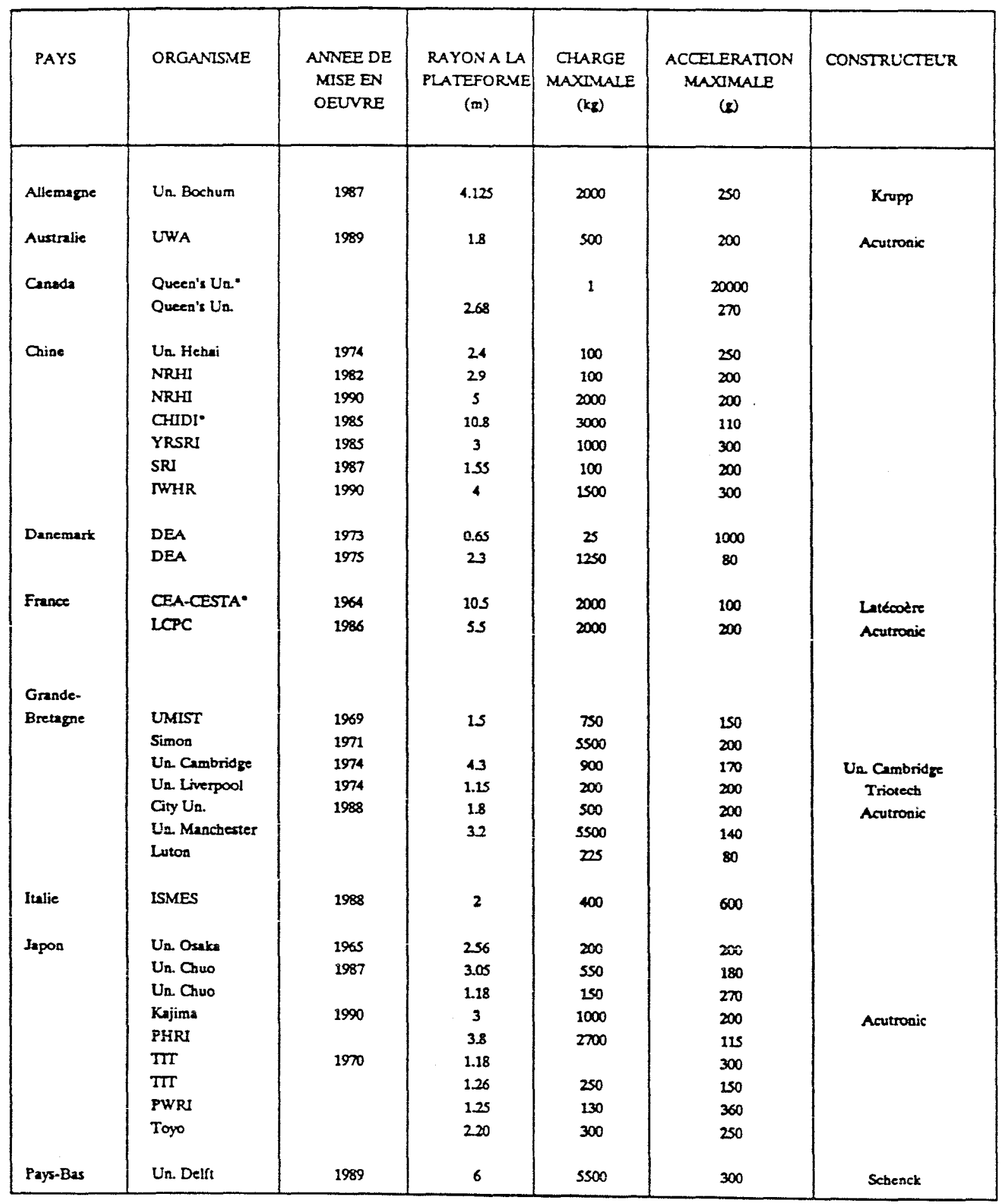




\begin{tabular}{|c|c|c|c|c|c|c|}
\hline PAYS & ORGANISME & $\begin{array}{l}\text { ANNEE DE } \\
\text { MISE EN } \\
\text { OEUVRE }\end{array}$ & $\begin{array}{c}\text { RAYONALA } \\
\text { PLATEFORME } \\
\text { (m) }\end{array}$ & $\begin{array}{l}\text { CHARGE } \\
\text { MAXIMALE } \\
\text { (kE) }\end{array}$ & $\begin{array}{l}\text { ACCEIERATION } \\
\text { MAXIMALE } \\
\text { (k) }\end{array}$ & CONSTRUCTEUR \\
\hline $\begin{array}{l}\text { URSS } \\
\text { USA }\end{array}$ & 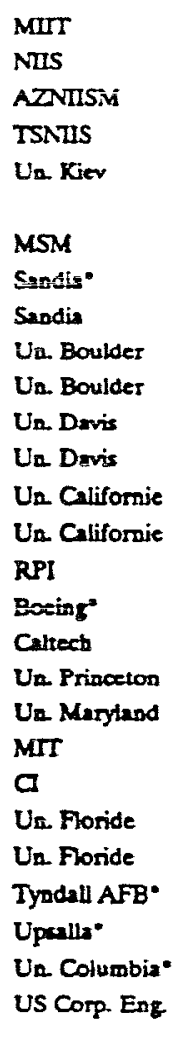 & $\begin{array}{l}1956 \\
1968 \\
1988 \\
1989 \\
1989 \\
1989\end{array}$ & $\begin{array}{l}25 \\
25 \\
55 \\
25 \\
2.5 \\
0.9 \\
75 \\
2.3 \\
5.49 \\
1.36 \\
9.1 \\
1 \\
\\
3 \\
1.3 \\
1 \\
1.83 \\
0.5 \\
65\end{array}$ & $\begin{array}{c}2 \times 10 \\
2 \times 170 \\
2500 \\
2200 \\
\\
\\
3500 \\
250 \\
2000 \\
136 \\
3600 \\
4000 \\
100 \\
1000 \\
200 \\
40 \\
100 \\
75 \\
75 \\
60 \\
50 \\
220 \\
10 \\
1 \\
6000\end{array}$ & $\begin{array}{l}320 \\
320 \\
500 \\
95 \\
300 \\
\\
200 \\
150 \\
200 \\
100 \\
300 \\
\\
100 \\
100 \\
200 \\
500 \\
175 \\
100 \\
200 \\
200 \\
\\
120 \\
100 \\
100 \\
4000 \\
2500 \\
350\end{array}$ & $\begin{array}{l}\text { Geniseo } \\
\text { Schaevitz } \\
\text { Acutronic } \\
\text { Boeing } \\
\text { Geniseo }\end{array}$ \\
\hline
\end{tabular}

* Installation non spécifque au génie civil. 